Therefore this form must be the required parametrical representation of any oval in tangential coordinates, if we choose the unit of length properly.*

SENDAI, JAPAN,

December, 1913.

\title{
ON THE CLASS OF DOUBLY TRANSITIVE GROUPS.
}

BY PROFESSOR W. A. MANNING.

(Read before the San Francisco Section of the American Mathematical Society, October 25, 1913.)

ThE class $u(u>3)$ of a doubly transitive group of degree $n$ is, according to Bochert, $\dagger$ greater than $\frac{1}{3} n-\frac{2}{3} \sqrt{n}$. If we confine our attention however to those doubly transitive groups in which one of the substitutions of lowest degree is of order 2, it appears that the class is greater than $\frac{1}{2} n-\frac{1}{2} \sqrt{n}$ -1 . The proof of this statement rests essentially upon the following

Lemma. The degree of a diedral group of class $u$ generated by two non-commutative substitutions of order 2 and degree $u$ is at most $\frac{3}{2} u$.

Let $s$ and $t$ be the two substitutions in question, and let the order of their product be $N=2^{a} p_{1}{ }^{a_{1}} p_{2}{ }^{a_{2}} \cdots p_{n}{ }^{a_{n}}$, where $p_{1}$, $p_{2}, \cdots$ are distinct odd primes. The transitive constituents of $\{s, t\}$ may be arranged as follows:

$s$ has $m_{1}$ cycles displacing letters not in $t$, and $t$ has $m_{2}$ cycles displacing letters not in $s$; there are $x_{i}$ regular constituents of order $X_{i}$, with a generator in both $s$ and $t$ (thus common cycles of $s$ and $t$ are explicitly included, while the preceding type of constituent of degree and order 2 is excluded); there are $y_{j}$ non-regular constituents of degree $Y_{j}$ and order $2 Y_{j}, Y_{j}$ an odd number; there are $y_{k}{ }^{\prime}$ non-regular constituents of degree $Y_{k}{ }^{\prime}$ and order $2 Y_{k}{ }^{\prime}, Y_{k}{ }^{\prime}$ even, with the generator of degree $Y_{k}{ }^{\prime}$ in $s$, and the generator of degree $Y_{k}^{\prime}-2$ in $t$; in like manner there are $y_{k}{ }^{\prime \prime}$ constituents of the order $Y_{k}{ }^{\prime}$ with $Y_{k}{ }^{\prime}-2$ letters in $s$ and $Y_{k}{ }^{\prime}$ letters in $t$. Since transitive

* Subsequently I have proved that an infinite number of cubes may be circumscribed about an ovoid body. The proof and application of this theorem will be published in the Science Reports of the Tôhoku University, Sendai, vol. 3 , no. 4 .

† Bochert, Math. Annalen, vol. 49 (1897), p. 131. 
diedral groups of degree $n$ are of class $n, n-1$, or $n-2$, and since in case the class is $n-2$ one generator of order 2 is odd and the other even, the above enumeration of possible constituents of $\{s, t\}$ is complete.

If $m_{1}$ or $m_{2}$ is zero the lemma is true as stated. Hence we assume that neither $m_{1}$ nor $m_{2}$ is zero and $N$, the order of the product $s t$, is even. The orders $\frac{1}{2} X_{i}$ run through all the divisors of $N$, unity and $N$ included, but any or all the multipliers $x_{i}$ may be zero. Let $\xi$ be written for $\Sigma x_{i} X_{i}$, where the summation extends to all the regular constituents of $\{s, t\}$ as agreed upon above. The numbers $Y_{j}$ are all the odd divisors of $N$, unity excepted, and the numbers $Y_{k}{ }^{\prime}$ are all the even divisors of $N$, including $N$ and excepting 2 . Some of the numbers $y_{j}, y_{k}{ }^{\prime}, y_{k}{ }^{\prime \prime}$ may be zero.

If now a census be taken of the transpositions in $s$ and $t$, there results

$$
\begin{aligned}
& 2 m_{1}+\xi+\Sigma y_{j}\left(Y_{j}-1\right)+\Sigma y_{k}{ }^{\prime} Y_{k}{ }^{\prime}+\Sigma y_{k}{ }^{\prime \prime}\left(Y_{k}{ }^{\prime}-2\right)=u, \\
& 2 m_{2}+\xi+\Sigma y_{j}\left(Y_{j}-1\right)+\Sigma y_{k}{ }^{\prime}\left(Y_{k}^{\prime}-2\right)+\Sigma y_{k}{ }^{\prime \prime} Y_{k}{ }^{\prime}=u,
\end{aligned}
$$

whence

$\left(m_{1}+m_{2}\right)=u-\xi-\Sigma y_{j}\left(Y_{j}-1\right)-\Sigma\left(y_{k}{ }^{\prime}+y_{k}{ }^{\prime \prime}\right)\left(Y_{k}{ }^{\prime}-1\right)$.

The degree of $\{s, t\}$ is

$$
2\left(m_{1}+m_{2}\right)+\xi+\Sigma y_{j} Y_{j}+\Sigma\left(y_{k}{ }^{\prime}+y_{k}{ }^{\prime \prime}\right) Y_{k}{ }^{\prime},
$$

or, when $2\left(m_{1}+m_{2}\right)$ is eliminated,

$$
2 u-\xi-\Sigma y_{j}\left(Y_{j}-2\right)-\Sigma\left(y_{k}{ }^{\prime}+y_{k}{ }^{\prime \prime}\right)\left(Y_{k}{ }^{\prime}-2\right) \text {. }
$$

If it be assumed that $N$ is divisible by $4,(s t)^{N / 2}$ is not identity, and its degree is not greater than

$$
\Sigma^{\prime \prime}\left(y_{k}{ }^{\prime}+y_{k}{ }^{\prime \prime}\right) Y_{k}{ }^{\prime}+\xi,
$$

where $\Sigma^{\prime \prime}$ extends the summation to all those constituents of $\{s, t\}$ whose order is a multiple of, or is, $2^{a+1}$. Because the class of $\{s, t\}$ is $u$,

$$
\Sigma^{\prime \prime}\left(y_{k}^{\prime}+y_{k}^{\prime \prime}\right) Y_{k}^{\prime}+\xi=u+h,
$$

where $h$ is a positive integer or zero. Now if $\Sigma^{\prime \prime}\left(y_{k}{ }^{\prime}+y_{k}{ }^{\prime \prime}\right) Y_{k^{\prime}}$ is zero by reason of $y_{k}{ }^{\prime}=0, y_{k}{ }^{\prime \prime}=0$, throughout the range of the summation $\Sigma^{\prime \prime}$, we eliminate $\xi$ from (1) by means of $\xi=u+h$ and obtain for the degree of $\{s, t\}$

$$
u-h-\Sigma y_{j}\left(Y_{j}-2\right)-\Sigma\left(y_{k}{ }^{\prime}+y_{k}{ }^{\prime \prime}\right)\left(Y_{k}{ }^{\prime}-2\right),
$$


a number less than $u$, which is absurd. Then $\{s, t\}$ has at least one non-regular constituent the degree of which is a multiple of, or is, $2^{a}$. Among these numbers let $Y_{0}^{\prime}$ be a minimum. We separate the non-regular constituents of degree $Y_{0}^{\prime}$ from the other constituents of $\{s, t\}$ and write

$$
\left(y_{0}^{\prime}+y_{0}^{\prime \prime}\right) Y_{0}^{\prime}+\Sigma^{\prime \prime \prime}\left(y_{k}{ }^{\prime}+y_{k}^{\prime \prime}\right) Y_{k}^{\prime}+\xi=u+h,
$$

whence

$$
y_{0}{ }^{\prime}+y_{0}^{\prime \prime}=\frac{u+h-\xi}{Y_{0}^{\prime}}-\Sigma^{\prime \prime \prime}\left(y_{k}{ }^{\prime}+y_{k}^{\prime \prime}\right) \frac{Y_{k}^{\prime}}{Y_{0}^{\prime}} .
$$

The $\Sigma^{\prime \prime \prime}$ denotes the exclusion of the $y_{0}{ }^{\prime}+y_{0}{ }^{\prime \prime}$ constituents of degree $Y_{0}^{\prime}$ and order $2 Y_{0}^{\prime}$ from the summation of nonregular constituents affected by $\Sigma^{\prime \prime}$. This value of $y_{0}{ }^{\prime}+y_{0}^{\prime \prime}$ if substituted in (1) gives, if $\Sigma^{\prime}=\Sigma-\Sigma^{\prime \prime}$,

$$
\begin{aligned}
& u\left(1+\frac{2}{Y_{0}^{\prime}}\right)-h\left(1-\frac{2}{Y_{0}^{\prime}}\right)-\frac{2 \xi}{Y_{0}^{\prime}}-\Sigma y_{j}\left(Y_{j}-2\right) \\
& -\Sigma^{\prime}\left(y_{k}{ }^{\prime}-y_{k}{ }^{\prime \prime}\right)\left(Y_{k}{ }^{\prime}-2\right)-2 \Sigma^{\prime \prime \prime}\left(y_{k}{ }^{\prime}+y_{k}{ }^{\prime \prime}\right)\left(\frac{Y_{k}^{\prime}}{Y_{0}^{\prime}}-1\right) .
\end{aligned}
$$

By reason of the assumption that $N$ is a multiple of 4 , the least value of $Y_{0}^{\prime}$ is 4, so that (2) is certainly not greater than $\frac{3}{2} u$.

Then $N$ is not divisible by 4 .

If $p$ is an odd prime divisor of $N$, consider $(s t)^{N / p}$, a substitution of degree $u$ or more. Its degree is at least

$$
y_{0} Y_{0}+\Sigma^{\prime \prime}\left(y_{k}{ }^{\prime}+y_{k}{ }^{\prime \prime}\right) Y_{k}{ }^{\prime}+\xi \text {, }
$$

where $Y_{0}=p^{a}$, the highest power of $p$ that divides $N$, and $\Sigma^{\prime \prime}$ extends to those non-regular constituents of which the degree is an even multiple of $p^{a}$. Just as in the preceding case the degree of $(s t)^{N / p}$ cannot reduce to $\xi$. But suppose that $y_{0}=0$. Then from the constituents under $\Sigma^{\prime \prime}$ select one of a minimum order $2 Y_{0}^{\prime}$, and write

$$
\Sigma^{\prime \prime \prime}\left(y_{k}{ }^{\prime}+y_{k}^{\prime \prime}\right) Y_{k}^{\prime}+\left(y_{0}^{\prime}+y_{0}^{\prime \prime}\right) Y_{0}^{\prime}+\xi=u+h .
$$

This leads again to (2), with this difference, that 6 is the least value we can assign to $Y_{0}{ }^{\prime}$. Then $y_{0}$ is not zero. Proceeding as before we get

$$
\left(Y_{0}-2\right) y_{0}=\left(1-\frac{2}{Y_{0}}\right)(u+h-\xi)-\Sigma^{\prime \prime}\left(y_{k}{ }^{\prime}+y_{k}{ }^{\prime \prime}\right) Y_{k}{ }^{\prime}\left(1-\frac{2}{Y_{0}}\right) \text {. }
$$


1914.] ON THE CLASS OF DOUBLY TRANSITIVE GROUPS. 471

Putting this in (1), we have

$$
\begin{aligned}
u(1 & \left.+\frac{2}{Y_{0}}\right)-h\left(1-\frac{2}{Y_{0}}\right)-\frac{2 \xi}{Y_{0}}-\Sigma^{\prime} y_{j}\left(Y_{j}-2\right) \\
& -\Sigma^{\prime}\left(y_{k}{ }^{\prime}+y_{k}^{\prime \prime}\right)\left(Y_{k}-2\right)-2 \Sigma^{\prime \prime}\left(y_{k}{ }^{\prime}+y_{k}{ }^{\prime \prime}\right)\left(\frac{Y_{k^{\prime}}}{Y_{0}}-1\right) .
\end{aligned}
$$

This number can exceed $\frac{3}{2} u$ only if $Y_{0}=3$. The lemma is proved in all cases except that in which $N=6$. In this case (1) becomes

$$
2 u-\xi-y-4\left(y^{\prime}+y^{\prime \prime}\right) .
$$

From $(s t)^{2}$ and $(s t)^{3}$ we have, respectively,

and

$$
\xi+3 y+6\left(y^{\prime}+y^{\prime \prime}\right)=u+h,
$$

while

$$
2\left(m_{1}+m_{2}\right)+\xi+6\left(y^{\prime}+y^{\prime \prime}\right)=u+k,
$$

$$
2\left(m_{1}+m_{2}\right)=2 u-2 \xi-4 y-10\left(y^{\prime}+y^{\prime \prime}\right) .
$$

By means of these three equations we may eliminate $y$ and $4\left(y^{\prime}+y^{\prime \prime}\right)$ from (1), and obtain the following expression for the degree of $\{s, t\}$ :

$$
\frac{1}{2} u-h-\frac{1}{2} k-\frac{1}{2} \xi \text {. }
$$

The proof of the lemma is now complete and we may use it in the proof of the following

THEOREM. The class $u(u>3)$ of a doubly transitive group of degree $n$ in which one of the substitutions of lowest degree is of order 2 is greater than $\frac{1}{2} n-\frac{1}{2} \sqrt{n}-1$.

There is by hypothesis in our doubly transitive group $G$ of class $u$ a substitution $s$ of order 2 and degree $u$. This substitution is one of a set of $w$ conjugates, and $w$ is greater than unity. Because of the double transitivity of $G$, every possible transposition that can be formed with $n$ letters is found among the $w$ substitutions of this set, and one as often as any other.* In fact, a given transposition occurs exactly $w u / n(n-1)$ times in the complete set of conjugates. There are $u(n-u)$ different transpositions possible in which one letter is displaced by $s$ and the other is one of the $n-u$ letters left fixed by $s$. No substitution that contains one of these transpositions is commutative with $s$, so that, if $y$ is

* Cf. Bochert, l. c., throughout this proof. 
472 ON THE CLASS OF DOUBLY TRANSITIVE GROUPS. [June,

the number of substitutions conjugate to $s$ which are not commutative with $s$,

$$
y \geqq \frac{2 w u(n-u)}{n(n-1)} .
$$

Each conjugate of $s$ displaces $u$ letters which may be associated in $\frac{1}{2} u(u-1)$ distinct pairs. Then the $w$ conjugates exhibit $w u$ letters and $\frac{1}{2} w u(u-1)$ pairs. There are $w u / n$ substitutions with a given letter in common and $w u(u-1) / n(n-1)$ substitutions with a given pair of letters in common. Hence the $u$ letters of $s$ are displaced $w u^{2} / n$ times, and the $\frac{1}{2} u(u-1)$ pairs of letters in $s$ are found in substitutions of the set of conjugates in all $w u^{2}(u-1)^{2} / 2 n(n-1)$ times.

Now let $m$ be the number of letters any substitution of the set has in common with $s$. Then if we sum for all the $w$ conjugates

$$
\Sigma_{w} m=\frac{w u^{2}}{n}, \quad \frac{1}{2} \Sigma_{w} m(m-1)=\frac{1}{2} \frac{w u^{2}(u-1)^{2}}{n(n-1)} .
$$

And by the lemma,

$$
\Sigma_{y} m \geqq \frac{1}{2} y u .
$$

An arithmetic relation between the above numbers may be set up by means of the identity

$$
\Sigma l^{2}=\Sigma\left(l-\frac{1}{k} \Sigma l\right)^{2}+\frac{1}{k}(\Sigma l)^{2},
$$

where the quantities $l_{1}, l_{2}, \ldots, l_{k}$, to which the summation is extended are any real numbers. This also implies

$$
\Sigma l^{2} \geqq \frac{1}{k}(\Sigma l)^{2} .
$$

Since doubly transitive groups of class $\frac{1}{2} n$ are known, it may be assumed once for all that the groups under discussion satisfy the condition

$$
u<\frac{1}{2} n \text {. }
$$

Then $y$ is not zero. Nor is $w-y$ zero, for $s$ is not included among the $y$ substitutions non-commutative with it. Now

$$
\Sigma_{w} m^{2}=\Sigma\left(m-\frac{u^{2}}{n}\right)^{2}+\frac{1}{w}\left(\Sigma_{w} m\right)^{2},
$$


1914.] ON THE CLASS OF DOUBLY TRANSITIVE GROUPS. 473 and hence

$\Sigma_{w}\left(m-\frac{u^{2}}{n}\right)^{2}=\Sigma_{w} m(m-1)+\Sigma_{w} m-\frac{1}{w}\left(\Sigma_{w} m\right)^{2}$ $=\frac{w u^{2}(u-1)^{2}}{n^{2}(n-1)}$.

And since

Again

$$
\begin{gathered}
\Sigma_{y}\left(m-\frac{u^{2}}{n}\right)^{2} \geqq y\left(\frac{u}{2}-\frac{u^{2}}{n}\right)^{2}, \\
\Sigma_{w-y}\left(m-\frac{u^{2}}{n}\right)^{2} \leqq \frac{w u^{2}(u-1)^{2}}{n^{2}(n-1)}-y\left(\frac{u}{2}-\frac{u^{2}}{n}\right)^{2} .
\end{gathered}
$$

$\Sigma_{w-y}\left(m-\frac{u^{2}}{n}\right)^{2} \geqq \frac{1}{w-y}\left(\Sigma_{w-y}\left(m-\frac{u^{2}}{n}\right)\right)^{2}$

$$
=\frac{1}{w-y}\left(\Sigma_{y} m-y \frac{u^{2}}{n}\right)^{2} \geqq \frac{1}{w-y} \frac{y^{2} u^{2}}{n^{2}}\left(\frac{n}{2}-u\right)^{2} .
$$

Hence

$$
\frac{w u^{2}(u-1)^{2}}{n^{2}(n-1)}-y\left(\frac{u}{2}-\frac{u^{2}}{n}\right)^{2} \geqq \frac{1}{w-y} \frac{y^{2} u^{2}}{n^{2}}\left(\frac{n}{2}-u\right)^{2},
$$

that is,

But

$$
\left(\frac{w}{y}-1\right) \frac{(n-u)^{2}}{n-1} \geqq\left(\frac{n}{2}-u\right)^{2} \text {. }
$$

whence finally,

$$
\frac{w}{y} \leqq \frac{n(n-1)}{2 u(n-u)},
$$

$$
\frac{n(n-u)}{u}-\frac{(n-u)^{2}}{n-1} \geqq\left(\frac{n}{2}-u\right)^{2} .
$$

Since the left-hand member of this inequality has, for $u<\frac{1}{2} n$, a negative derivative

$$
-2\left(\frac{n}{2 u}\right)^{2}+\frac{n-u}{n-1},
$$

it is a decreasing function of $u$, as $u$ increases from 4 to $\frac{1}{2} u$. Then from a known lower limit of $u$ another may be calculated by substitution for $u$ of the known limit on the left of the sign 
474 ON THE Class OF DOUBLY TRANSITIVE GRoups. [June, of inequality. For example, it is known that $u$ is greater than $\frac{1}{4} n,{ }^{*}$ whence

and therefore

$$
\left(\frac{n}{2}-u\right)^{2} \leqq \frac{15}{16} n-\frac{9}{16} \frac{n}{n-1},
$$

$$
u>\frac{1}{2} n-\frac{1}{2} \sqrt{a_{1} n},
$$

if $a_{1}$ is put for $15 / 4$. This limit may in turn be used to find another of the same form. Let us assume

$$
u>\frac{1}{2} n-\frac{1}{2} \sqrt{a_{k} n}
$$

and seek a recurrence formula for $\sqrt{a_{k}}$. We get

$$
\left(\frac{n}{2}-u\right)^{2} \leqq \frac{n}{4}\left(1+\frac{2 \sqrt{a_{k}}}{\sqrt{n}-\sqrt{a_{k}}}+\frac{a_{k}-1}{n-1} \frac{\sqrt{n}+\sqrt{a_{k}}}{\sqrt{n}-\sqrt{a_{k}}}\right),
$$

or since

where

$$
\begin{gathered}
\frac{a_{k}}{\left(\sqrt{n}-\sqrt{a_{k}}\right)^{2}}>\frac{a_{k}-1}{n-1} \frac{\sqrt{n}+\sqrt{a_{k}}}{\sqrt{n}-\sqrt{a_{k}},} \\
u>\frac{1}{2} n-\frac{1}{2} \sqrt{n a_{k+1}},
\end{gathered}
$$

$$
\sqrt{a_{k+1}}=\frac{\sqrt{n}}{\sqrt{n}-\sqrt{a_{k}}}=\frac{\sqrt{n}}{\sqrt{n}}-\frac{\sqrt{n}}{\sqrt{n}}-\cdots-\frac{\sqrt{n}}{\sqrt{n}}-\frac{\sqrt{a_{1}}}{1} .
$$

Here $a_{1}=15 / 4$, and the number of component fractions is $k+1$. We may now write $\sqrt{a_{k+1}}$ as the quotient of two continuants:

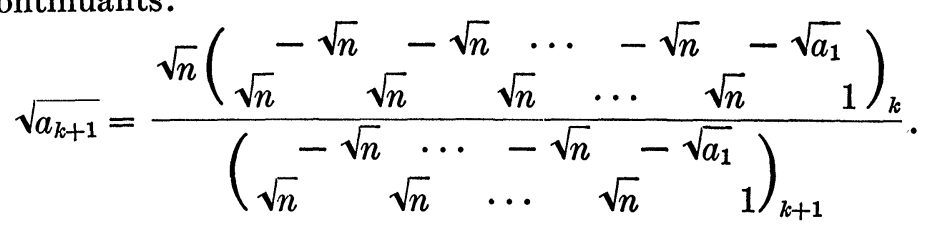

Let $C_{k}$ denote the continuant of the $k$ th order

$$
\left(\begin{array}{cccc}
-\sqrt{n} & -\sqrt{n} & \cdots & -\sqrt{n} \\
\sqrt{n} & \sqrt{n} & \cdots & \sqrt{n}
\end{array}\right) .
$$

* Bochert, Math. Annalen, vol. 40 (1892), p. 182. 
1914.] ON THE CLASS OF DOUBLY TRANSITIVE GROUPS. 475

Then

and $C_{k}=\sqrt{n}\left(C_{k-1}-C_{k-2}\right)$.

$$
\sqrt{a_{k+1}}=\sqrt{n} \frac{C_{k-1}-\sqrt{a_{1}} C_{k-2}}{C_{k}-\sqrt{a_{1}} C_{k-1}},
$$

This difference equation has the solution

$$
C_{k}=\frac{\alpha^{k+1}-\beta^{k+1}}{\alpha-\beta},
$$

where $\alpha+\beta=\sqrt{n}, \alpha \beta=\sqrt{n}$, and in consequence

Then

$$
\alpha=\frac{1}{2} \sqrt{n}+\frac{1}{2} \sqrt{n-4 \sqrt{n}} \text {. }
$$

$$
\sqrt{a_{k+1}}=\sqrt{n} \frac{\left(\alpha^{k}-\beta^{k}\right)-\sqrt{a_{1}}\left(\alpha^{k-1}-\beta^{k-1}\right)}{\left(\alpha^{k+1}-\beta^{k+1}\right)-\sqrt{a_{1}}\left(\alpha^{k}-\beta^{k}\right)} .
$$

As $k$ approaches infinity, this fraction approaches the limiting value $\beta=\frac{1}{2} \sqrt{n}-\frac{1}{2} \sqrt{n-4 \sqrt{n}}$. Then finally

$$
u>\frac{n}{4}(1+\sqrt{1-4 / \sqrt{n}}) \text {. }
$$

To call attention to the remarkable restriction imposed upon the class of $G$ by this formula a few pairs of values of $n$ and $u$ calculated from it are given:

\begin{tabular}{l|rrrrrrrrrrrrrr}
$n=\mid 21$ & 22 & 23 & 24 & 25 & 26 & 27 & 28 & 29 & 30 & 31 & 32 & 33 & 34 \\
\hline$u>$ & 6 & 6 & 8 & 8 & 8 & 8 & 8 & 10 & 10 & 10 & 10 & 12 & 12 & 12 \\
\hline$n=$ & 35 & 36 & 37 & 38 & 39 & 40 & 41 & 42 & 43 & 44 & 45 & 46 & 47 & 48 \\
\hline$u>$ & 12 & 14 & 14 & 14 & 14 & 16 & 16 & 16 & 16 & 16 & 18 & 18 & 18 & 18 \\
\hline \hline
\end{tabular}

In this connection it should perhaps be noted that there exists a doubly transitive group* of degree 28 and class 12 with 63 conjugate substitutions of order 2 and degree 12 in it. In this set of conjugates there are exactly 32 substitutions noncommutative with a given substitution of the set, and with which each of them has exactly 6 letters in common. There are 30 substitutions that have just 4 letters in common with the given substitution and are commutative with it, making up the total number of conjugates. This appears to indicate

* Manning, Amer. Jour. of Math., vol. 35 (1913), p. 258. 
that this limit for $u$ cannot be much increased. But if a limit of simpler form is desired one may use

$$
u>\frac{1}{2} n-\frac{1}{2} \sqrt{n}-1 \text {. }
$$

If $n$ is greater than 22, this number is less than $\frac{1}{4} n(1$ $+\sqrt{1-4 / \sqrt{n}})$; in fact the latter approaches $\frac{1}{2} n-\frac{1}{2} \sqrt{n}-\frac{1}{2}$ as $n$ increases. If $n$ is less than 23 it is known that this limit holds, for all primitive groups of class less than 14 are known,* and the classes 4 and 6 , which alone are in question here, belong to no primitive groups of higher degree than 10 .

STANFORD UNIVERSITY, October, 1913.

\section{CHRISTOFFEL'S MATHEMATICAL WORKS.}

E. B. Christoffel, Gesammelte mathematische Abhandlungen, Unter Mitwirkung von A. KRAZER und G. FABER, herausgegeben von L. Maurer. Zwei Bände. B. G. Teubner, Leipzig, 1910.

WHEN one turns over the pages of the collected works of a mathematician such as this one, arranged in chronological order, and notes the varied fields in which the author worked, he feels an impulse to follow the methods of his literary colleagues and to try to find the influences which played upon the author. To what extent was he influenced by direct contact with other masters? Or perhaps was he that year lecturing upon a certain subject and thus was naturally led to an attempt to solve some of its problems? These and other questions arise in the mind of a reviewer, and he must decide whether he shall amuse himself chasing fancies or turn to the more serious task of the kind of a review such as we are accustomed to expect.

In the present instance some of the former questions receive a partial answer as he reads the interesting biography of the author written by Dr. C. F. Geiser for the thirty-fourth volume of the Mathematische Annalen and reprinted at the beginning of the first volume now under discussion. Here one reads that in his student days at Berlin Christoffel came under the influence of Dirichlet, Borchardt, and Steiner, and later, ences.

* Cf. Manning, Amer. Jour. of Math., vol. 35 (1913), p. 229, for refer- 